\title{
Band Shifts of IR Spectra of Aromatic Thiols Adsorbed on Gold Electrodes with Potential Changes
}

\author{
Takeshi SASAKI* and Tatsuo ISHIKAWA
}

Received February 12, 1993 ; Accepted March 29, 1993

\section{INTRODUCTION}

Recently numerous in-situ spectroscopic studies on molecules adsorbed on electrode surfaces have been reported. In-situ infrared spectroscopy gives fundamental information on the vibrational characteristics of adsorbed molecules at electrode-electrolyte interfaces. The analysis of carefully measured spectra can elucidate the characteristics of interaction between the adsorbed molecules and electrode systems. This report is concerned with the effect of electrode potential on the adsorbed molecules. The band shifts with electrode potential of aromatic thiols adsorbed on gold polycrystalline electrodes were investigated by in-situ IRAS experiments.

\section{EXPERIMENTAL}

The experimental apparatus and measurement procedure used in this report are basically the same as those reported elsewhere. 1,2)

The test electrode was an Au plate (99.998, Johnson Mattey Co.) and the counter electrode was a Pt wire. The potential was referred to an $\mathrm{Ag}-\mathrm{AgCl}$ electrode. The organic materials investigated in this report were p-mercaptophenol (MP) and 4-methoxybenzenethiol (MBT) of reagent grade which were used as received (Wako Pure Chem. Inc. and Aldrich Chem. Co., respectively).

After cleaning the electrode by cycling potential, the electrode was contacted with $10 \mathrm{mM} \mathrm{MP}$ in $0.1 \mathrm{M} \mathrm{HClO}_{4}$ or $10 \mathrm{mM}$ MBT in 4:1 methyl alcohol and

Department of Metallurgical Engineering, Faculty of Engineering, Hokkaido University (Sapporo, 060 Japan)

Key Words: in-situ IRAS, gold electrode, aromatic thiol, band shift water solution to adsorb the organic materials, and after removing unadsorbed organic materials from the system, measurements of spectra were carried out by changing electrode potential.

The FTIR spectrometer employed was a JASCO FTIR/8000 with an MCT detector. Interferograms of $500-2000$ scans were collected and co-added at each potential to improve the $\mathrm{S} / \mathrm{N}$ ratio. The spectra were recorded in terms of absorbance.

\section{RESULTS AND DISCUSSION}

It has been clarified from the spectroscopic and electrochemical investigations ${ }^{2)}$ that MP molecules adsorb to Au electrodes with the molecular plane nearly perpendicular to the electrode surface forming a mercaptide bond between $\mathrm{S}$ and $\mathrm{Au}$.

The variation in spectra in the potential region of $0-550 \mathrm{mV}$, where no chemical reactions occur, is presented in Fig. 1. The spectrum acquired

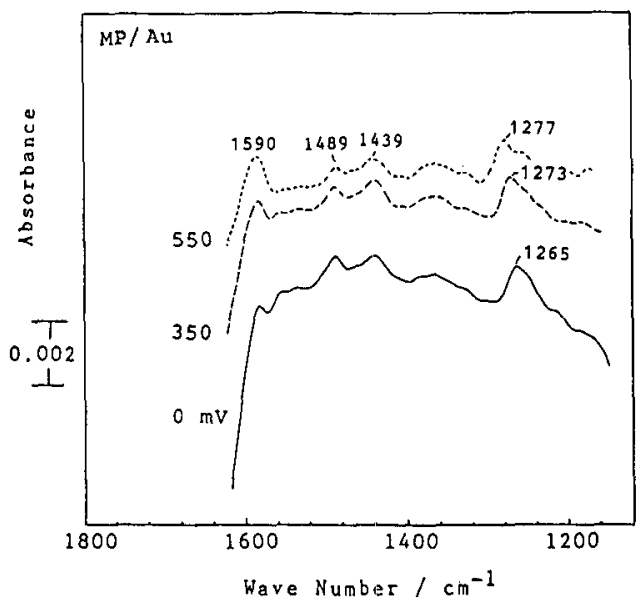

F1g. I Potential variation in spectra of $\mathbb{M}$ adsorbed on a gold electrode. The reference spectrum was acquired at $750 \mathrm{mV}$. 
at $750 \mathrm{mV}$, where most of the MP changed into the oxidized state, was used as the reference. Three bands at 1590, 1489, and $1439 \mathrm{~cm}^{-1}$ are assigned to $\mathrm{C}=\mathrm{C}$ skeletal deformation of the benzene ring. The peaks at around $1270 \mathrm{~cm}^{-1} \mathrm{cor}-$ respond to the $\mathrm{C}-\mathrm{O}$ stretching vibration.

The figure shows that the bands from C-O stretching vibration shifted to high frequencies with the increase in potential, whereas few shifts were observed in the bands from the skeletal deformation. The plot of $\mathrm{C}-\mathrm{O}$ vibrational frequency against electrode potential shows that the shift linearly depended on the applied potential with a gradient of $22 \mathrm{~cm}^{-1} \mathrm{~V}^{-1}$.

Similar experiments on MBT indicated that MBT adsorbed on the Au electrode was stable in the potential range of 0 $800 \mathrm{mV}$. The spectral changes of MBT in the potential range are shown in Fig. 2, where the spectrum for the oxidized state of MBT at $1100 \mathrm{mV}$ was taken the reference. The bands at 1590 and 1489 $\mathrm{cm}^{-1}$ were attributed to the skeletal

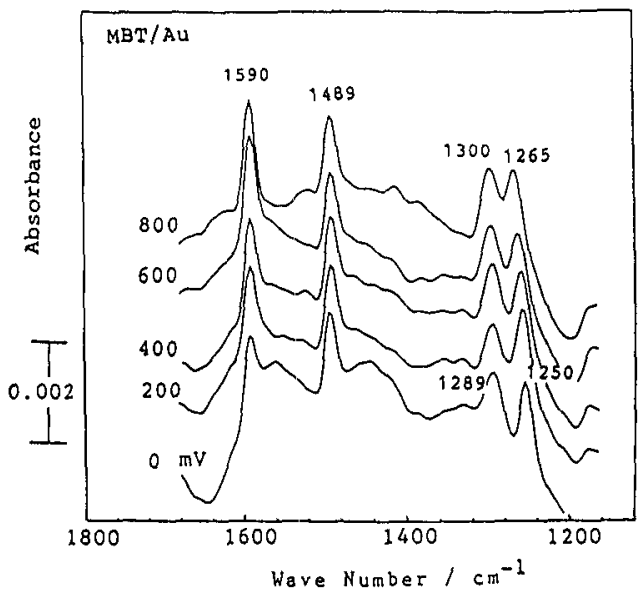

Fig. 2 Potential variation in spectra of MBT adsorbed on a gold electrode. The reference spectrum was acquired at $1100 \mathrm{mV}$.

deformation of the benzene ring and this indicates that MBI is also adsorbed on the Au surfaces with the molecular surface nearly perpendicular to the electrode surface. The band at around $1260 \mathrm{~cm}^{-1}$ is due to asymmetry stretching vibration of $\left.\mathrm{C}-\mathrm{O}-\mathrm{C}^{3}\right)$. The figure shows that the skeletal vibration of the benzene ring is almost unaffected by the change in the electrode potential, but the C-O-C mode shifts linearly to higher wavenumber by $19 \mathrm{~cm}^{-1} \mathrm{v}^{-1}$. The peak at around $1290 \mathrm{~cm}^{-1}$ could not be assigned and the peak shifted slightly, by $5 \mathrm{~cm}^{-1}$ with potential change of $1 \mathrm{~V}$.

Many experimental and theoretical studies on the shifts in vibrational frequency of carbon monoxide adsorbed on Pt electrodes have been reported ${ }^{4}$. The C-O vibrational frequency linearly shifts with potential changes, and this is explained in terms of stark effect due to electrostatic potential changes at the electric double layer or back donation of electrons to the $\pi$-antibonding from the electrode. The reasons for the band shifts are still under investigation.

The experimental results on $M P$ and MBT showed that $\mathrm{C}-\mathrm{O}$ and $\mathrm{C}-\mathrm{O}-\mathrm{C}$ vibrational frequencies shifted with potential changes, though the skeletal vibrational frequencies depended little on them. This frequency behavior demonstrates that the effects of electrode potential changes appeared stronger on the bonds farther from the electrode surface and may provide a clue to the reason for the band shift mentioned above. In any case, studies on the band shift of adsorbed organic molecules with electrode potential have just begun and further experimental and theoretical investigations are needed.

This work was supported in part by a grant-in-aid from Asahi Glass Foundation.

\section{REFERENCES}

1) T. Sasaki, I.T. Bae, D.A. Scherson, B.G. Bravo and M.P. Soriaga, Langmuir, 6, 1234(1990)

2) T. Sasaki and T. Ishikawa, J. Surf. Fin. Soc. Japan, 43, 457(1992)

3) R.M. Silverstein, G.C. Bassler and T.C. Morril, Spectrometric Identification of Organic Compounds, 4th ed. , p108, (John Willy \& Sons, 1981)

4) For example, B. Beden, A. Bewick, K. Kunimatsu and $\mathrm{J}$. Lamy, Electroanal. Chem., 142,345(1982), C. Korzeniewski and S. Pons, J. Chem. Phys., 85, 4153(1986) 